\title{
Bend loss fiber optics design based on sinusoidal and ellipse configurations
}

\author{
NoOr AzIE Azura MOHD ARIF ${ }^{*}$, Dilla Durhya BURHANUDDIN ${ }^{2}$, \\ SAHBUDIN SHAARI ${ }^{2}$, ABANG ANNUAR EHSAN ${ }^{2}$ \\ ${ }^{1}$ Centre for Pre-University Studies, Universiti Malaysia Sarawak, \\ 94300 Kota Samarahan, Sarawak, Malaysia \\ ${ }^{2}$ Institute of Microengineering and Nanoelectronics (IMEN), \\ Universiti Kebangsaan Malaysia (UKM), \\ 43600 Bangi, Malaysia \\ *Corresponding author: manaazura@unimas.my, aaehsan@ukm.edu.my
}

\begin{abstract}
Bending losses in optical fibers comprise one of the extrinsic attenuations that contribute to optical loss and they are essential for optical fiber bending sensor applications. This work investigated the optical loss in a standard single-mode step-index fiber optics due to fiber bending at $1550 \mathrm{~nm}$ wavelength. Variations in macro-bending loss with curvature radius and turn number have been measured. Curvature radius and turn number are examined for sinusoidal and elliptical shaped bending configurations. It has been found that the loss increases as the bending radius and number of turns increase. The result also showed that elliptical shaped bending configuration produced more loss in contrast to that of sinusoidal shaped at bending angles of $180^{\circ}$ and $360^{\circ}$. The study on the macro-bending loss in terms of curvature radius and turn number for both elliptical and sinusoidal shaped bending configurations is beneficial for future fiber optic sensor applications.
\end{abstract}

Keywords: sinusoidal shape, ellipse shape, bending loss, number of turns, curvature radius.

\section{Introduction}

Light loss mechanism in an optical fiber can be related to the transmission, reflection and refraction of light [1]. Attenuation mechanism can be grouped into two attenuation categories, namely intrinsic and extrinsic attenuation. Substances that are present in the fiber optics as impurities contribute to intrinsic attenuation. Imperfection in the glass is another example that contributes to intrinsic attenuation. Both examples are caused by the properties of the fiber and these impurities and imperfection can be solved with a more accessible technique compared with extrinsic attenuation. Extrinsic attenuation is caused by external forces [2] ]. Macro- and micro-bending are techniques in which loss can be affected by one of these techniques. Generally, losses in an optical fiber are not desired for telecommunication applications but it can be valuable for optical fiber bending sensor applications. Macro- and micro-bending designs are design configurations that can provide effective loss control mechanism in fiber bending sen- 
sors. Numerous fiber bending sensors have been proposed to measure different physical parameters such as voltage, pressure, strain, temperature [ $\underline{3}-\underline{5}]$. At present, the bending loss mechanism is being used in wearable sensors for respiration and heartbeat detection.

Micro-bend loss is due to microscopic fiber deformation in the core-cladding interface in which bending is caused by a small radius of curvature $[\underline{2}, \underline{6}]$. Macro-bends are bends on the fiber with the radius of the curve being much higher than the radius of the fiber, which can be denoted as $r \gg a$ where $a$ is the core radius and $r$ is the radius of curvature. Commonly, macro-bends can be described by a bend angle and bend radius. Basically, light is not confined and guided in the fiber optics core when the fiber is bent. Total internal reflection of light propagating in the core at the core-cladding interface will fail when light propagates at an angle higher than the critical angle. Nowadays, several approaches to bending loss have been explained using different fiber bending configurations, including single loop, U-shape, sinusoidal and figure-of-eight shapes [1]. In this article, the study on the relationships between macro-bending loss and bending radius and between macro-bending loss and turn number for sinusoidal and elliptical bending configurations is conducted. Different radius of curvature and turn numbers have been considered to investigate their effect on bending loss.

\section{Theoretical study}

A single-mode with length $l$, and general bending loss $L$ for a single-mode is usually obtained by $[\underline{1}, \underline{6}-\underline{10}]$

$$
L_{\mathrm{s}}=10 \log \left(e^{2 \alpha l}\right)=8.686 \alpha L
$$

where $\alpha$ is the bending loss coefficient, and it is a function of bending radius, light wavelength and optical fiber material and structure.

\subsection{Bending radius}

According to $[\underline{6}, \underline{8}]$, the bending loss with various bending radii $R$ is

$$
L_{R}=\eta_{R_{1}} \exp \left(-\eta_{R_{2}} R\right)
$$

where $\eta_{R_{1}}$ and $\eta_{R_{2}}$ are fitting parameters. This formula was generated using fixed parameters, namely, bending angle $\theta$, wrapping turn $N$ and wavelength $\lambda$.

\subsection{Turns number}

Bending loss with different turn numbers at the same value of wavelength can simplify the relationship as a linear equation

$$
L_{N}=\eta_{N} N
$$

where $L_{N}$ is the loss due to the number of turns $N$. In addition to being simple, this linear equation is practical and thus is suitable and valid for a larger radius of curvature $[\underline{6}, \underline{8}]$. 


\subsection{Configuration of fiber bending}

Guidance of light traveling through standard single-mode fiber SMF-28e+ can be related and discussed using the phenomenon of total internal reflection (TIR). Although, ray-tracing analysis usually is used when the size of the fiber or waveguide region is much larger than the wavelength which is more practical in multimode fiber optics. However, this theory is used only proves that the loss between two configurations of fiber optics design. The propagation of light in half-turn of ellipse, sinusoidal and ellipse shapes are discussed.

\subsubsection{Propagation of light for the $U$-shape or half turn of the ellipse shape}

In the sensing region, with U-shape configuration (Fig. 1), the angle of a ray with the normal to the core-cladding interface should be greater than the critical angle. With this situation, the ray is capable of propagating through the sensing part. If $\theta$ represents the angle that a guided ray makes with the normal to the core-cladding interface in the straight fiber, then the corresponding angle $\varphi$ at the outer surface of the bent-core is given by the expression done by [11-13]:

$$
\sin \varphi=\frac{B+h}{B+w} \sin \theta
$$

where $h$ is the distance at which the ray is incident on the entrance of fiber from the core-cladding boundary $B$ is the bending radius, and $w$ is the waveguide width. Similarly, angle $\delta$ of the ray at the inner surface of the bent-core is given by:

$$
\sin \delta=\frac{B+h}{B} \sin \theta
$$

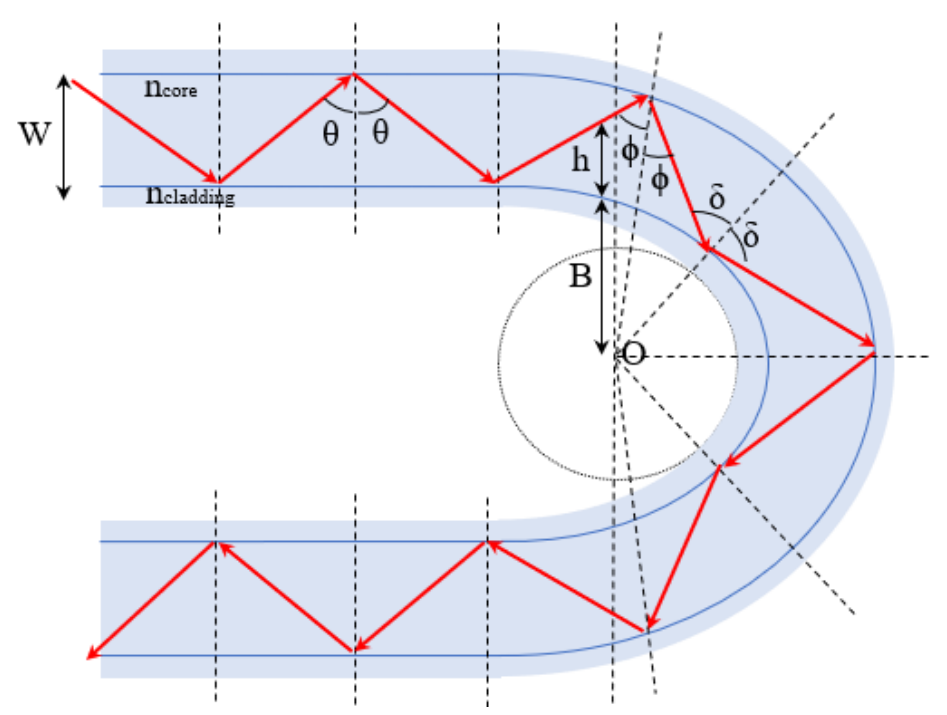

Fig. 1. Meridional ray in U-shaped or half-turn of ellipse shape sensing region. 
Thus, the angle $\theta$ is transformed to angle $\varphi$ and $\delta$. Hence, $\varphi_{1}$ and $\varphi_{2}$ for the outer surface can be written as:

$$
\varphi_{1}=\sin ^{-1}\left[\frac{(B+h) n_{\text {cladding }}}{(B+w) n_{\text {core }}}\right] \text { and } \quad \varphi_{2}=\sin ^{-1}\left[\frac{B+h}{B+w}\right]
$$

Similarly, for the inner surface of the sensing region it can be written as:

$$
\delta_{1}=\sin ^{-1}\left[\frac{(B+h) n_{\text {cladding }}}{B n_{\text {core }}}\right] \text { and } \delta_{2}=90^{\circ}
$$

\subsubsection{Propagation of light for the sinusoidal shape}

Sinusoidal shapes can be explained by adding one more U-shape but in the opposite direction $\left(180^{\circ}\right)$ as shown in Fig. 2 . The angles at the first bend can be explained by Eqs. (6) and (7). This explanation was done by [13] but at S-shaped waveguide. Hence,

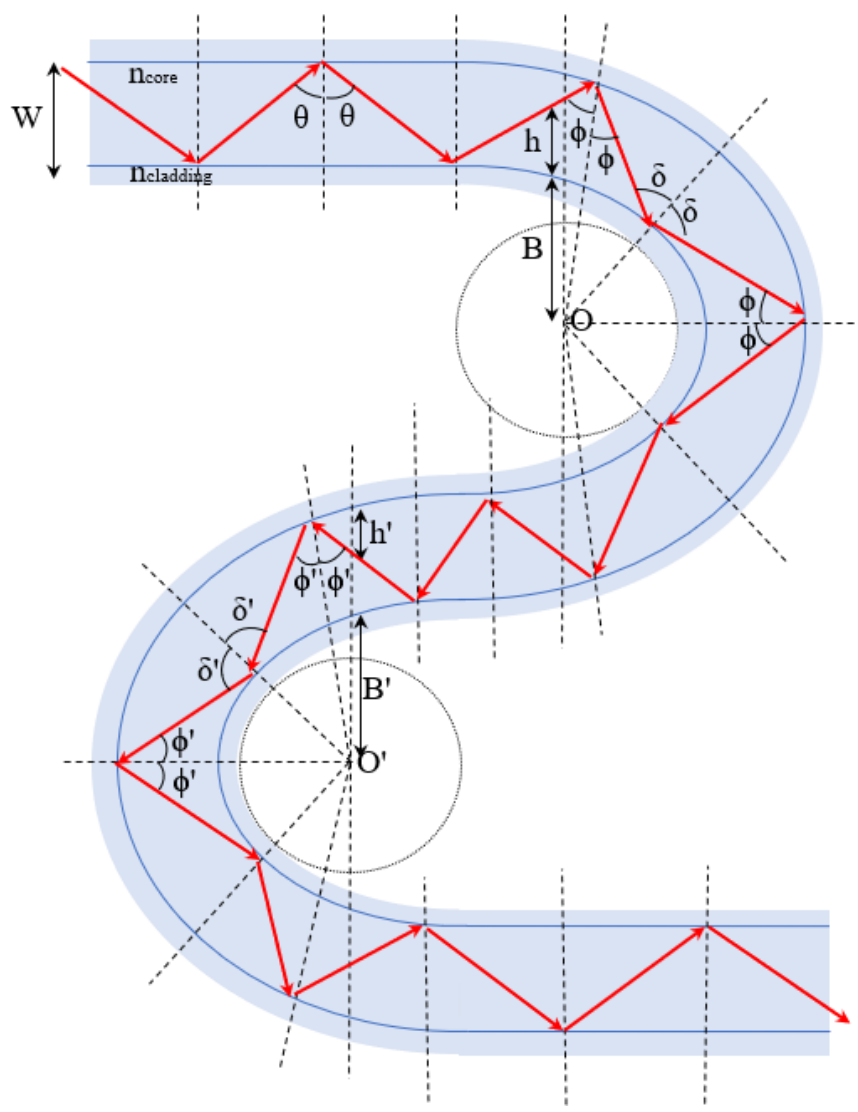

Fig. 2. Meridional ray in the sinusoidal shape sensing region. 
by understanding the way of calculation, the range of the angle of incidence at the first bend changes the angle at the second bend, and it can be written as:

$$
\begin{aligned}
& \varphi_{1}^{\prime}=\sin ^{-1}\left[\frac{B+w}{B^{\prime}+w} \frac{\left(B^{\prime}+w-h^{\prime}\right) n_{\text {cladding }}}{\left(B^{\prime}+w\right) n_{\text {core }}}\right] \\
& \varphi_{2}^{\prime}=\sin ^{-1}\left[\frac{B+w}{B^{\prime}+w} \frac{B^{\prime}+w-h^{\prime}}{B^{\prime}+w}\right]
\end{aligned}
$$

Then,

$$
\begin{aligned}
& \delta_{1}^{\prime}=\sin ^{-1}\left[\frac{B^{\prime}+w}{B^{\prime}} \frac{B+w}{B^{\prime}+w} \frac{\left(B^{\prime}+w-h^{\prime}\right) n_{\text {cladding }}}{\left(B^{\prime}+w\right) n_{\text {core }}}\right] \\
& \delta_{2}^{\prime}=\sin ^{-1}\left[\frac{B^{\prime}+w}{B^{\prime}} \frac{B+w}{B^{\prime}+w} \frac{B^{\prime}+w-h^{\prime}}{B^{\prime}+w}\right]
\end{aligned}
$$

where $w$ is the waveguide width, $B^{\prime}$ is the bending radius of the second bend and $h^{\prime}$ is the ray's entrance height from the first bend to the second bend.

\subsubsection{Propagation of light for an ellipse shape}

The range of the angle of incidence at the first bend in an ellipse shape, as shown in Fig. 3, can be explained by previous equations that are modified by [13] for the spiral waveguide. Furthermore, the range of the angle of incidence with the normal to the core-cladding interface at the first bend changes and values of the angle of incidence at the second bend can be calculated as follows:

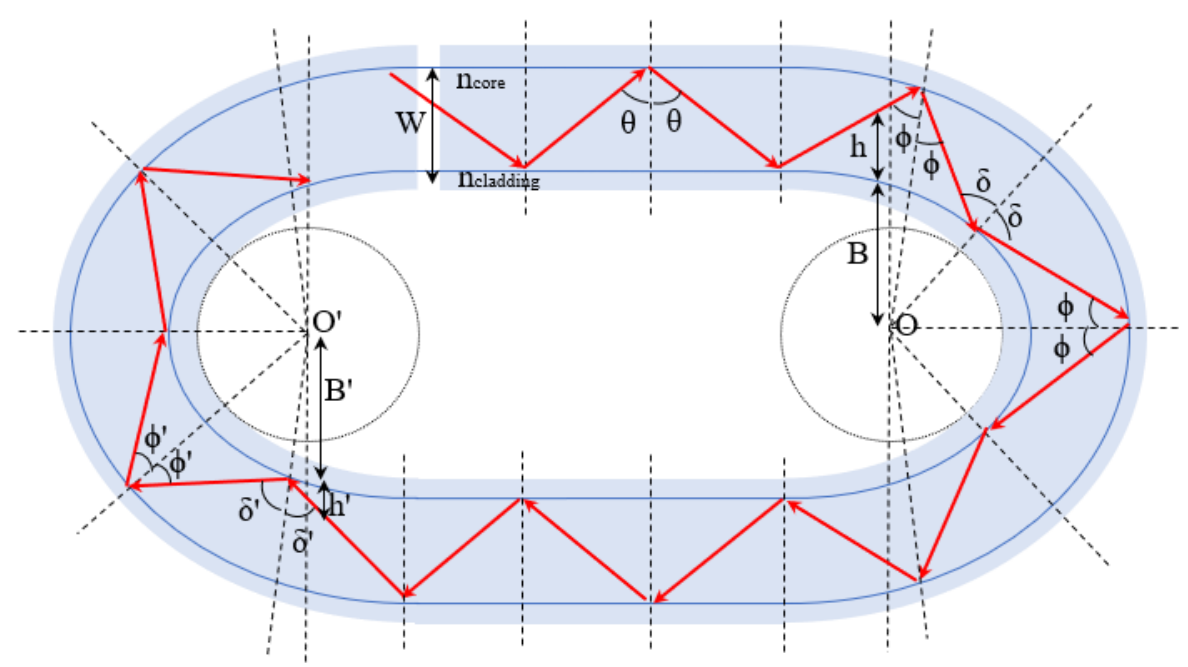

Fig. 3. Meridional ray in ellipse shape sensing region. 


$$
\begin{aligned}
& \delta_{1}^{\prime}=\sin ^{-1}\left[\frac{B+w}{B+h^{\prime}} \frac{\left(B^{\prime}+h^{\prime}\right) n_{\text {cladding }}}{B^{\prime} n_{\text {core }}}\right] \\
& \delta_{2}^{\prime}=\sin ^{-1}\left[\frac{B+w}{B+h^{\prime}} \frac{B^{\prime}+h^{\prime}}{B^{\prime}}\right]
\end{aligned}
$$

then,

$$
\begin{aligned}
& \varphi_{1}^{\prime}=\sin ^{-1}\left[\frac{B^{\prime}}{B^{\prime}+w} \frac{B+w}{B+h^{\prime}} \frac{\left(B^{\prime}+h^{\prime}\right) n_{\text {cladding }}}{B^{\prime} n_{\text {core }}}\right] \\
& \varphi_{2}^{\prime}=\sin ^{-1}\left[\frac{B^{\prime}}{B^{\prime}+w} \frac{B+w}{B+h^{\prime}} \frac{B^{\prime}+h^{\prime}}{B^{\prime}}\right]
\end{aligned}
$$

Owing to the configuration of the bending fiber, the partial loss of these propagating rays at the outer $\left(L_{\mathrm{o}}\right)$ or inner $\left(L_{\mathrm{i}}\right)$ bend surface due to each of the TIRs is as follows:

$$
L_{\mathrm{o}}=\frac{4\left(\psi / \theta_{\mathrm{cc}}^{2}\right)}{\sqrt{\theta_{\mathrm{cc}}^{2}-\psi^{2}}}\left(\frac{\lambda \alpha}{4 \pi n_{2}}\right) \quad \text { or } \quad L_{\mathrm{i}}=\frac{4\left(\psi / \theta_{\mathrm{cc}}^{2}\right)}{\sqrt{\theta_{\mathrm{cc}}^{2}-\psi^{2}}}\left(\frac{\lambda \alpha}{4 \pi n_{2}}\right)
$$

where $L_{\mathrm{o}}$ or $L_{\mathrm{i}}$ is the partial loss of optical power, $\lambda$ is the wavelength, $\alpha$ is the bulk-attenuation coefficient at the region of contact, $n_{2}$ is the refractive index at the boundary and $\theta_{\mathrm{cc}}$ is the critical angle, where

$$
\theta_{\mathrm{cc}}=\frac{\pi}{2}-\sin ^{-1}\left(\frac{n_{2}}{n_{1}}\right)=\cos ^{-1}\left(\frac{n_{2}}{n_{1}}\right)
$$

and $\psi=90^{\circ}-\varphi$ or $\psi=90^{\circ}-\delta, \varphi$ and $\delta$ being the angle of incidence of light on the interface at the bent outer and inner sensing regions. Then, the number of ray reflections per unit length at each outer $\left(N_{\mathrm{o}}\right)$ or inner $\left(N_{\mathrm{i}}\right)$ bend surface of the sensing region can be written as:

$$
N_{\mathrm{o}}=\frac{\cot \varphi}{2 w} \quad \text { or } \quad N_{\mathrm{i}}=\frac{\cot \delta}{2 w}
$$

Hence, the loss of optical power per unit length $L_{N}$ can be determined by multiplying the corresponding $L$ and $N$ values for the inner and outer bend surfaces,

$$
L_{N}=L_{\mathrm{o}} \times N_{\mathrm{o}} \quad \text { or } \quad L_{N}=L_{\mathrm{i}} \times N_{\mathrm{i}}
$$

\section{Method}

In this experiment, measurements were conducted using standard single-mode optical fiber (Corning SMF-28e+) with core and cladding diameters of 9 and $125 \mu \mathrm{m}$, respectively. Considering the refractive index of core and cladding to be 1.4645 and 1.456, respectively. The optical fiber of the sinusoidal shape of different vertices as shown 
Ta b l e 1. Fiber optics configurations with detailed parameters.

\begin{tabular}{llll}
\hline Shape & Wavelength $[\mathrm{nm}]$ & Tip vertex $a[\mathrm{~cm}]$ & Lateral vertex $b[\mathrm{~cm}]$ \\
\hline & & 2.5 & 0.75 \\
Sinusoidal/ & \multirow{2}{*}{1550} & 2.0 & 0.75 \\
ellipse & 1.5 & 0.75 \\
& & 1.0 & 0.75 \\
\hline
\end{tabular}

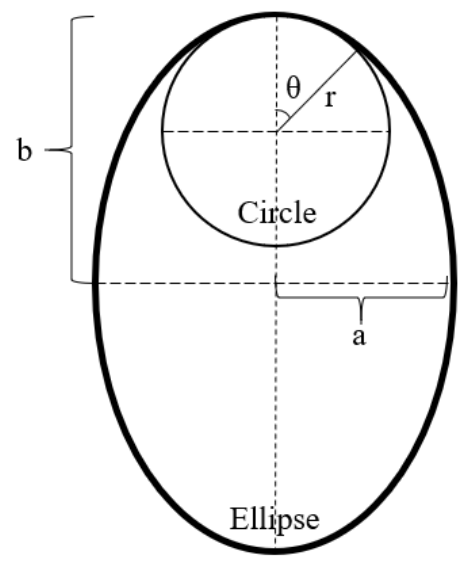

Fig. 4. Radius $r$ of the circle in an ellipse with tip vertex $a$ and lateral vertex $b$ of ellipse.

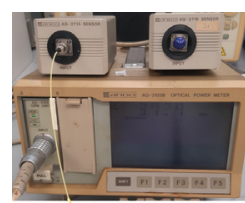

Optical power meter
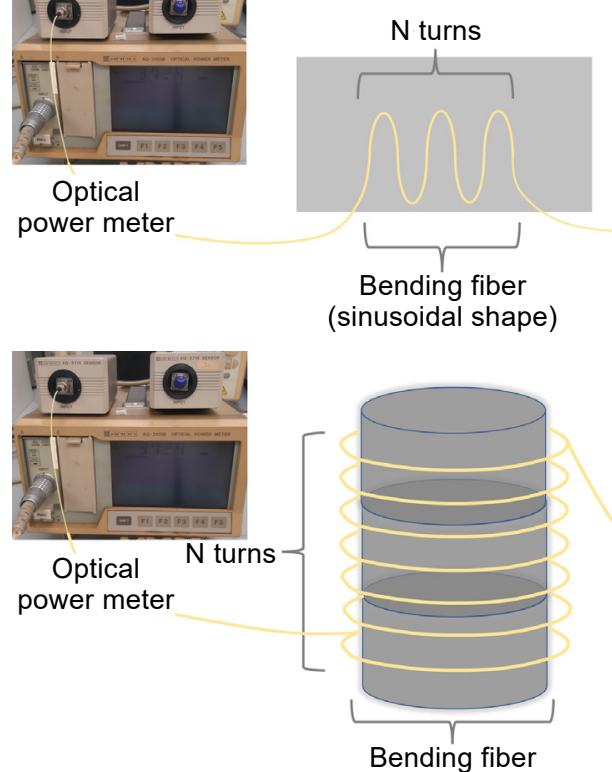

(sinusoidal shape)

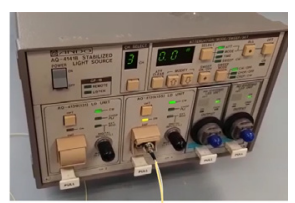

Wavelength tunable light output $(1550 \mathrm{~nm})$

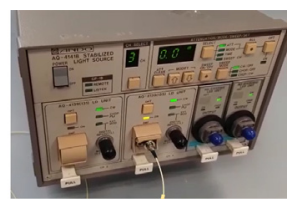

Wavelength tunable light output $(1550 \mathrm{~nm})$

Fig. 5. Schematic diagram of the optical set-up for measuring bending loss of the fiber. 
in Table 1 was made and used for the bent fiber with the input of the power meter operating at the wavelength of $1550 \mathrm{~nm}$. An ellipse shape was bent with a double sinusoidal shape, which can be explained in Fig. 4. To study the effect of the number of turns $(N)$ on bending loss, up to 6 turns were investigated. A schematic diagram of the set-up for measuring the bending loss is shown in Fig. 5.

\section{Results and discussion}

For investigating the essential characterization of bending loss, the effect of bending radius $R$ was studied and then the influence of turns number $N$ on the loss was investigated.

\subsection{Effect of bending radius}

When the fiber is bent at less than $0.15 \mathrm{~cm}$, the bending loss will increase rapidly, as shown in Fig. 6. Thus, it is observed that a larger bending radius will induce a higher
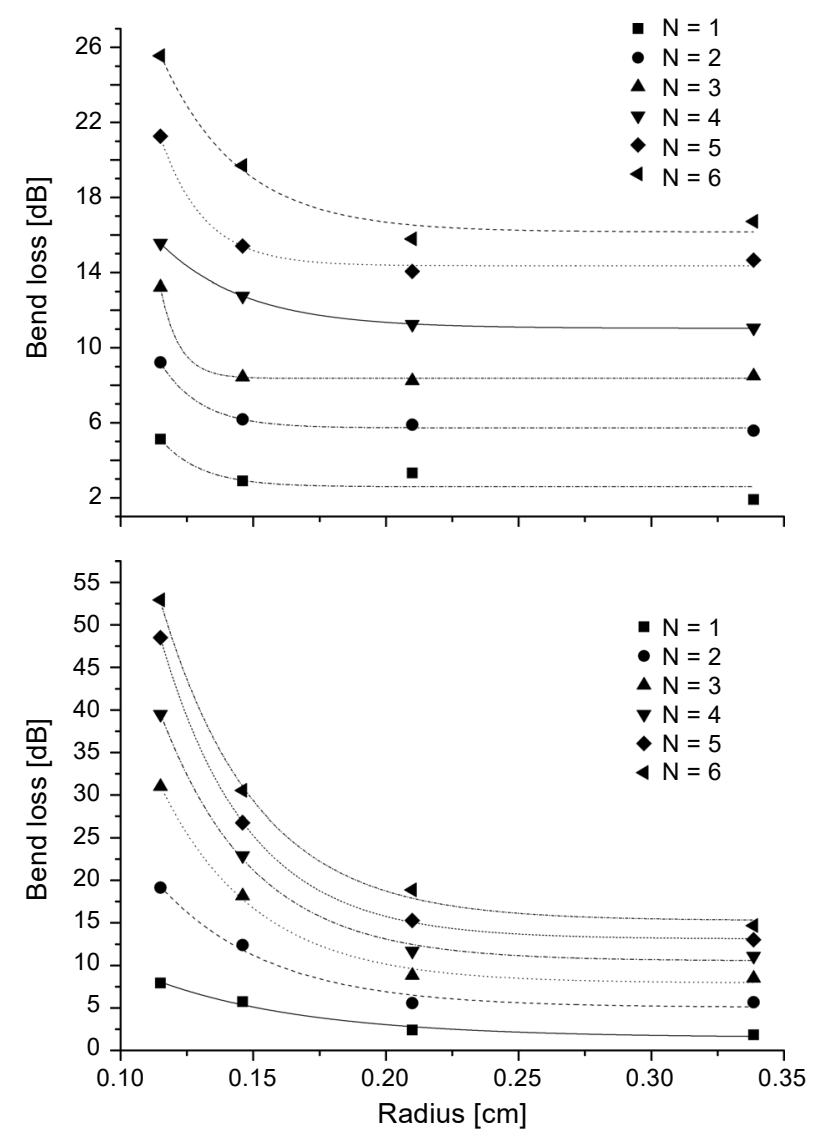

b

Fig. 6. Relationship between bending radius and bending loss: (a) sinusoidal shape, and (b) ellipse shape. 
bending loss. The variation shows an exponentially varying curve that satisfies the proposed relationship between losses and curvature radius by $[\underline{14}, 15]$. Tip and lateral vertices effect the bending radius. The radius of the circle within the ellipse can be obtained according to the general formula of the ellipse base.

\subsection{Influence of turns number}

The bending losses from 1 to 6 turns in $1550 \mathrm{~nm}$ wavelength with various bending radiuses are measured in this section. The experimental results, as shown in Fig. 7, show that the relationship between bending loss $L_{N}$ and turn number $N$ can be simplified as a linear equation for both fiber configurations satisfying the proposed relationship between losses and number of turns in $[\underline{2}, 14]$. By considering and comparison with the general single-mode bending loss $[\underline{1}, \underline{6}, \underline{7}]$, the following relationships can be determined:

$$
B=8.686 \alpha l
$$

And $l$ can be calculated using

$$
\begin{aligned}
& l=\frac{\pi}{2}[3(a+b)-\sqrt{3(a+b)(a+3 b)}] \text { for a sinusoidal shape, and } \\
& l=\pi[3(a+b)-\sqrt{3(a+b)(a+3 b)}] \text { for an ellipse shape }
\end{aligned}
$$

where $a$ and $b$ are tip vertex and lateral vertex of an ellipse, respectively.

As can be seen from Fig. 7, variations of $L$ against $N$ plotted for both configurations show that the lower bend loss occured when the lateral vertex of sinusoidal shape is $2.5 \mathrm{~cm}$. Both values of semi-axes of the ellipse are essential to determining the sensitivity of fiber optics on the bend loss effect. Ellipse is considered in this situation be-

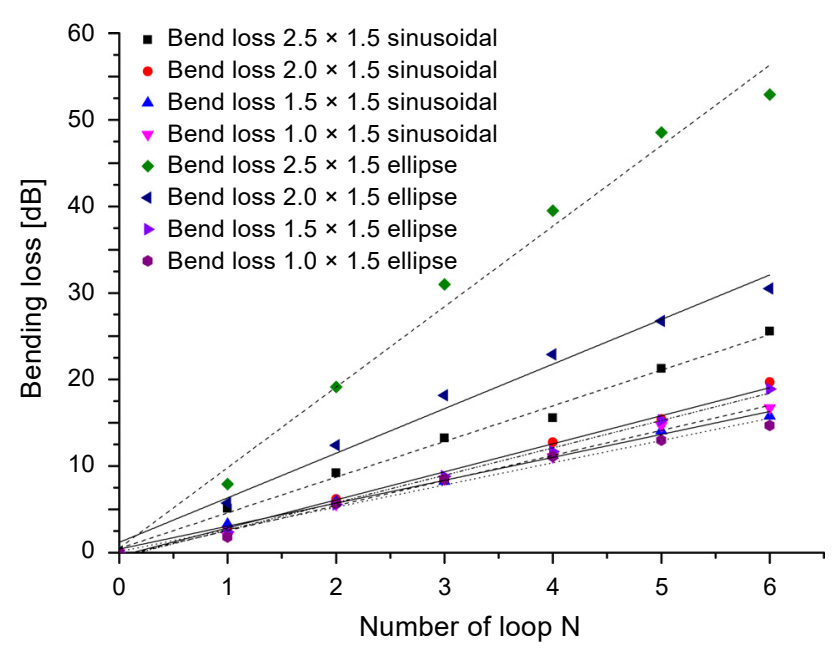

Fig. 7. Variation of bending loss versus a number of turns: (a) sinusoidal shape, and (b) ellipse shape. 
$\mathrm{T} \mathrm{a} \mathrm{b} \mathrm{l} \mathrm{e} \mathrm{2.} \mathrm{Sensitivity} \mathrm{according} \mathrm{to} \mathrm{changes} \mathrm{in} \mathrm{loop.}$

\begin{tabular}{lll}
\hline Tip vertex $a[\mathrm{~cm}]$ & Lateral vertex $b[\mathrm{~cm}]$ & Sensitivity \\
\hline 2.5 & 0.75 & 1.8257 \\
2.0 & 0.75 & 1.6330 \\
1.5 & 0.75 & 1.4142 \\
1.0 & 0.75 & 1.1547 \\
\hline
\end{tabular}

$\mathrm{T}$ a b $1 \mathrm{e}$ 3. Loss due to different refractive indexes.

\begin{tabular}{llll}
\hline$n_{\text {core }}$ & $n_{\text {cladding }}$ & Loss in sinusoidal shape [W] & Loss in ellipse shape [W] \\
\hline 1.45000 & 1.44470 & $3.40 \times 10^{-7}$ & $3.25 \times 10^{-6}$ \\
1.46770 & 1.46280 & $3.83 \times 10^{-7}$ & $3.20 \times 10^{-6}$ \\
1.44940 & 1.44400 & $3.36 \times 10^{-7}$ & $3.26 \times 10^{-6}$ \\
1.46165 & 1.45600 & $3.37 \times 10^{-7}$ & $3.23 \times 10^{-6}$ \\
1.46145 & 1.45600 & $3.34 \times 10^{-7}$ & $3.30 \times 10^{-6}$ \\
\hline
\end{tabular}

cause the changes of amplitude and period on sinusoidal shape affect the geometry of the bend-like elliptical shape. As seen in Table 2, a change in loop periodicity is inversely proportional to changes in loop amplitudes. The most sensitive sensing region has a more significant parameter of sensor sensing sensitivity, and the sensitivity $S$ can be related as

$$
S=\sqrt{\frac{a}{b}}
$$

where $a$ and $b$ are the tip and lateral vertices of an ellipse, respectively. This sensitivity can be applied for both shapes due to the relation shape of the fiber optic design.

\subsection{Influence of refractive index}

Figures 6 and 7, indicated that losses are higher in elliptical shape configuration. This phenomenon can be related to the behaviour of light propagation into the sensing region due to fiber bending, as discussed in the theoretical part that is influenced by a fiber optic refractive index. The loss of optical power was calculated at the fixed values of $w, \varepsilon, \lambda$ and $\theta_{\mathrm{cc}}$ using Eq. (19). The values of power loss for sinusoidal and elliptical shapes of fiber optics configurations are $3.34 \times 10^{-7}$ and $3.23 \times 10^{-6} \mathrm{~W}$, respectively. This result shows that elliptical shape fiber optics configuration generated higher loss than that of sinusoidal shape fiber optics configuration. Different values of core and cladding of the refractive index were examined as shown in Table 3 and it confirmed that higher loss was generated in ellipse shape due to the total internal reflection.

\section{Conclusion}

The effect of bending radius and turn number on bending loss in step-index fiber optics has been investigated. This study showed that bending loss decreases as curvature ra- 
dius and turns number decrease. Furthermore, the type of configurations of fiber bending, either a sinusoidal or elliptical shape also affects the bending loss. Elliptical shape generated higher loss than did sinusoidal shape. By analyzing the relationship between bending loss and bending radius and turn number, it is found that a single-mode optical fiber has potential in fiber optics sensor application due to higher sensitivity on bending loss.

Acknowledgements - Author are thankful to Nanophotonics Lab, Institute of Microengineering and Nanoelectronics (IMEN), UKM for providing facilities. Financial support from the Ministry of Higher Education (MOHE) and Universiti Malaysia Sarawak are gratefully acknowledged.

\section{References}

[1] Mohd Arif N.A.A, Berhanuddin D.D., Ehsan A.A., Design parameters of fiber-optics bend for sensing applications, International Journal of Nanoelectronics and Materials 13, 2020, pp. 107-112.

[2] Amanu A.A., Macro bending losses in single mode step index fiber, Advances in Applied Sciences 1(1), 2016, pp. 1-6, DOI: $10.11648 /$ j.aas.20160101.11.

[3] Cheng Y., Wang R., Sun J., Gao L., A stretchable and highly sensitive graphene-based fiber for sensing tensile strain, bending, and torsion, Advanced Materials 27(45), 2015, pp. 7365-7371, DOI: 10.1002/adma.201503558.

[4] Di H., XIN Y., Sun S., Electric current measurement using fiber-optic curvature sensor, Optics and Lasers in Engineering 77, 2016, pp. 26-30, DOI: 10.1016/j.optlaseng.2015.07.009.

[5] Satriananda D., Sugesti E.S., Muayyadi A.A., Analysis of voltage sensor based on optical fiber macrobending structure using piezoelectric for 1500-1600 $\mathrm{nm}$ wavelength, e-Proceeding of Engineering 4(3), 2017, pp. 4206-4209.

[6] Zendehnam A., Mirzaei M., Farashiani A., Horabadi Farahani L., Investigation of bending loss in a single-mode optical fibre, Pramana - Journal of Physics 74(4), 2010, pp. 591-603, DOI: $10.1007 /$ s12043-010-0052-5.

[7] Wang Q., Farrell G., Freir T., Theoretical and experimental investigations of macro-bend losses for standard single mode fibers, Optics Express 13(12), 2005, pp. 4476-4484, DOI: 10.1364 / opex.13.004476.

[8] Nandwalkar J.R., Pete D.J., Amelioration in measurement of macro bend loss for optical fiber retrospection, [In] Proceedings of International Conference on Wireless Communication. Lecture Notes on Data Engineering and Communications Technologies, [Eds.] Vasudevan H., Gajic Z., Deshmukh A., Vol. 36, Springer, Singapore, 2020, pp. 417-428, DOI: 10.1007/978-981-15-1002-1_43.

[9] Waluyo T. B., Bayuwati D., Mulyanto I., The effect of macro-bending on power confinement factor in single mode fibers, Journal of Physics: Conference Series 985, 2018, article 012001, DOI: 10.1088/1742-6596/985/1/012001.

[10] Qiu J., Zheng D., Zhu K., FANG B., Cheng L., Optical fiber sensor experimental research based on the theory of bending loss applied to monitoring differential settlement at the earth-rock junction, Journal of Sensors, Vol. 2015, 2015, article 346807, DOI: 10.1155/2015/346807.

[11] Gupta B.D., Dodeja H., TomaR A.K., Fibre-optic evanescent field absorption sensor based on a U-shaped probe, Optical and Quantum Electronics 28(11), 1996, pp. 1629-1639, DOI: $10.1007 /$ BF00331053.

[12] Khijwania S.K., SRinivasan K.L., Singh J.P., Performance optimized optical fiber sensor for humidity measurement, Optical Engineering 44(3), 2005, article 034401, DOI: 10.1117/1.1870753.

[13] Prabhakar A., Mishra N., Verma D., Mukherji S., Investigation of dual-bend serpentine/spiral waveguides coupled to a microchannel system for competent, evanescent-wave-absorption-based, 
on-chip, biological-/chemical-sensing applications, RSC Advances 8(62), 2018, pp. 35539-35550, DOI: $10.1039 / \mathrm{C} 8 \mathrm{RA} 06527 \mathrm{~F}$.

[14] Tsao S.L., Cheng W.M., Simplified formula of bending loss for optical fiber sensors, Fiber and Integrated Optics 21(5), 2002, pp. 333-344, DOI: 10.1080/01468030290087723.

[15] Mahdi W.H., Murad F.A., Al-Zamilly A.F., Studying the effect of curvature in the multimode optical fiber and calculate the critical radius of curvature for the wavelength $850 \mathrm{~nm}$ and $1550 \mathrm{~nm}$, Journal of Engineering and Applied Sciences 13(18), 2018, pp. 7662-7664, DOI: 10.3923/jeasci. $\underline{2018.7662 .7664 .}$

[16] Mohd Arif N.A.A., EhSAn A.A., Sensitivity optimization of U-shaped fiber optics based on the Taguchi method, OSA Continuum 4(7), 2021, pp. 2024-2035, DOI: 10.1364/OSAC.430129.

Received April 22, 2020 in revised form July 20, 2020 